\title{
Absolute polar duty cycle division multiplexing over wavelength division multiplexing system
}

\begin{abstract}
The performance of absolute polar duty cycle division multiplexing (AP-DCDM) over wavelength division multiplexing (WDM) system is presented based on the simulation results. The AP-DCDM signal has narrower bandwidth than conventional time division multiplexing (TDM) signal, which makes its implementation in WDM system advantageous. In this paper, characteristics of AP-DCDM and TDM signals in WDM system are compared at the speed of $40 \mathrm{Gbit} / \mathrm{s}$ per channel, for the minimum allowed channel spacing and the chromatic dispersion tolerance. The results clearly show that AP-DCDM performs significantly better than TDM. By using AP-DCDM, $1.28 \mathrm{Tbit} / \mathrm{s}(32 \times 40 \mathrm{Gbit} / \mathrm{s})$ was successfully transmitted over $320 \mathrm{~km}$ standard single mode fiber. Spectral efficiency of $0.64 \mathrm{~b} / \mathrm{s} / \mathrm{Hz}$ was achieved by using $10 \mathrm{Gbit} / \mathrm{s}$ transmitters and receivers without polarization multiplexing.
\end{abstract}

Keyword: Optical fiber communication; Absolute polar duty cycle division multiplexing; Wavelength division multiplexing; Dispersion 